\title{
ARTICLE Amyloid single-cell cytotoxicity assays by nanomotion detection
}

\author{
Francesco S Ruggeri ${ }^{1,4}$, Anne-Laure Mahul-Mellier ${ }^{2}$, Sandor Kasas ${ }^{1}$, Hilal A Lashuel ${ }^{2}$, Giovanni Longo ${ }^{1,3}$ and Giovanni Dietler $^{1}$
}

Cells are extremely complex systems able to actively modify their metabolism and behavior in response to environmental conditions and stimuli such as pathogenic agents or drugs. The comprehension of these responses is central to understand the molecular bases of human pathologies, including amyloid misfolding diseases. Conventional bulk biological assays are limited by intrinsic cellular heterogeneity in gene, protein and metabolite expression, and can investigate only indirectly cellular reactions in non-physiological conditions. Here we employ a label-free nanomotion sensor to study single neuroblastoma cells exposed to extracellular monomeric and amyloid $a$-synuclein species in real-time and in physiological conditions. Combining this technique with fluorescence microscopy, we demonstrate multispecies cooperative cytotoxic effect of amyloids and aggregate-induced loss of cellular membrane integrity. Notably, the method can study cellular reactions and cytotoxicity an order of magnitude faster, and using 100-fold smaller volume of reagents when compared to conventional bulk analyses. This rapidity and sensitivity will allow testing novel pharmacological approaches to stop or delay a wide range of human diseases.

Cell Death Discovery (2017) 3, 17053; doi:10.1038/cddiscovery.2017.53; published online 21 August 2017

\section{INTRODUCTION}

The complexity of the brain architecture and its cellular heterogeneity are central limitations to evaluate the specific responses, biological functions and causes of cytotoxicity in living organisms when subjected to external stimuli such as the exposition to pathogenic agents or drugs. Conventional biological assays that investigate cellular modifications in bulk conditions are affected by an intrinsic variability, which can be explained by a heterogeneity in the expression levels of genes, proteins or metabolites in the living systems under consideration. ${ }^{1}$ These methodologies can evaluate cellular stress and vitality by using spectroscopic indirect techniques, exploiting fluorescent dyes or absorbance measurements. Thus, by only considering the average cellular reactions from a large population of cells, ${ }^{2,3}$ they might conceal important but subtle individual cellular responses. ${ }^{4}$ Furthermore, these bulk assays can assess mid- and long-term cellular responses generally arising long after the exposure to chemical or physical stimuli. For this reason, these methods could not account for a phenotypic modification in the studied cells during the assessment and they could mask the dynamics of early events that are important in the initiation and long-term propagation of cellular responses to this exposure.

The current lack of understanding of the molecular mechanisms involved in the onset of amyloid-related human diseases, such as Parkinson's disease (PD) ${ }^{5-7}$ is partially caused by this difficulty in studying the response of populations of cellular systems with intrinsic variability. In PD, the presynaptic protein $a$-synuclein (a-syn) accumulates in intracellular fibrillar aggregates, which are recognized as main pathological hallmarks of the disease., Recently, animal and cellular models have shown that $a$-syn can be released extracellularly and taken up by neighboring cells, where it can seed the aggregation of endogenous $a$-syn. ${ }^{10,11}$ Thus, a keen interest has aroused for the potential contribution of extracellular $a$-syn in the neurodegeneration and PD progression. ${ }^{12-14}$ Therefore, there is an urgent need for real-time assays that allow single-cell handling, without interference from neighboring cells, and that are able to overcome the intrinsic limitations of bulk assays.

Towards this goal, we developed a new experimental approach to study single-cell responses using an innovative nanomotion sensor $^{15}$ that can transduce in measurable fluctuations the nanoscale metabolically-related movements of cells. ${ }^{16}$ This methodology allows monitoring in real-time and at a single-cell scale the response to external stimuli in the order of hours, way before the conventional high-throughput bulk assays, providing a platform of unparalleled sensitivity and time resolution to monitor different biological systems, such as bacteria, yeast and mammalian cells with unprecedented resolution. ${ }^{15-18}$ Indeed, previous works have demonstrated how micron-sized nanomechanical sensors can transduce even the very small fluctuations produced by conformational changes in proteins and that the vibrations of just few proteins can produce a measurable fluctuation of the sensor. $^{19}$

Here we applied this nanomotion detector to study the specific responses of a neuron-model system exposed to physiological concentrations of extracellular monomeric and toxic amyloid aggregated species of $a$-syn. We used a dopaminergic human neuroblastoma cell line (M17) to evaluate directly the neurotoxic mechanisms and cellular responses of these cells upon exposure to different $a$-syn species, monomeric or aggregated, in order to

\footnotetext{
${ }^{1}$ Institute of Physics, Ecole Polytechnique Fédérale de Lausanne (EPFL), Lausanne CH-1015, Switzerland; ${ }^{2}$ Laboratory of Molecular and Chemical Biology of Neurodegeneration, Brain Mind Institute, Faculty of Life Sciences, École Polytechnique Fédérale de Lausanne (EPFL), Lausanne CH-1015, Switzerland and ${ }^{3}$ Istituto di Struttura della Materia, Consiglio Nazionale delle Ricerche, Rome, Italy.

Correspondence: G Longo (giovanni.longo@ism.cnr.it) or G Dietler (giovanni.dietler@epfl.ch)

${ }^{4}$ Current Address: Department of Chemistry, University of Cambridge, CB21EW, UK.

Received 6 April 2017; revised 6 June 2017; accepted 30 June 2017; Edited by N Barlev
} 

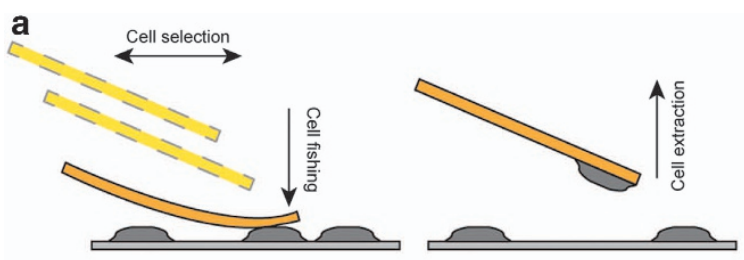

b
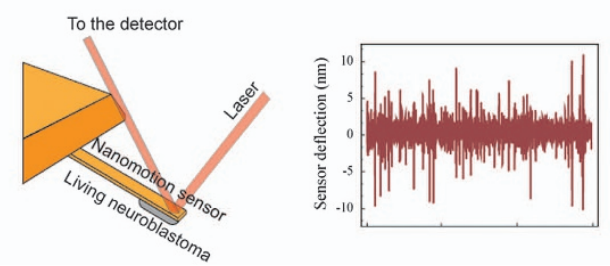

C

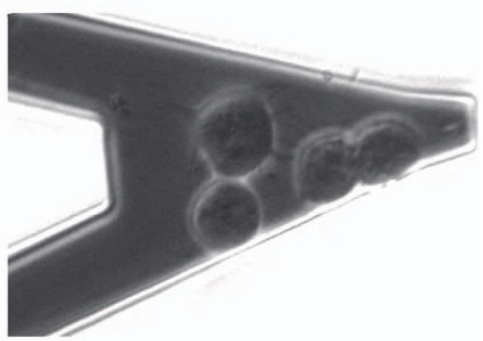

Figure 1. Schematics nanomotion sensor setup. (a) The functionalized cantilever is approached to a cell and gently pressed over it. The cell is immobilized on the surface of the sensor and the cantilever is retracted to allow the cell firm adhesion. (b) Conventional laser-based detection system of the vertical fluctuations of the sensor and resulting nanomotion signal of the cantilever deflection. (c) Optical image of four neuroblastoma cells adhering on the cantilever and linked by their neurites (Supplementary Movies S1-S3).

understand how these agents might influence cellular metabolism and viability and the role of aggregation in cellular toxicity. As a preliminary step, we studied the cytotoxicity of these species by conventional bulk culture studies. Next, we combined fluorescence microscopy with nanomotion investigations, to monitor in real-time the activity of the cells during the exposure to the $a$-syn species, with a particular focus on the conditions that produce cytotoxicity. Our analyses show that single-cell measurements yield new insights in the cellular response to amyloid species of a-syn.

Overall, its peculiarities make this an ideal innovative research platform to test new pharmacological methods to prevent or delay the formation of the toxic species on the aggregation pathway and to block neurodegeneration and the onset of amyloid-related pathologies.

\section{RESULTS}

Cytotoxicity studies

At first, we performed a complete characterization of the biophysical and structural properties of the recombinant $a$-syn monomeric and aggregated proteins, using bulk and singlemolecule techniques: Thioflavin T (ThT), Circular Dichroism (CD) and atomic force microscopy (AFM) imaging (Supplementary Information 1, Supplementary Figure S1). ${ }^{20}$ In our cytotoxicity studies, we considered at the following $a$-syn preparations: (a) freshly filtrated monomers, (b) a solution of mature fibrillar aggregates and (c) a stable mixture of oligomeric and mature fibrillar species, which we called crude mixture. ${ }^{20-22}$ Then, we assessed their toxicity towards human dopaminergic neuroblastoma M17 cells in culture. We exposed the M17 cells to monomeric, fibrillar or to the crude mixture of $a$-syn. After 4 days of treatment, we collected the M17 cells and stained them with Propidium lodide (PI), a vital dye that enters only in cells with disrupted plasma membrane. The percentage of PI-positive cells (dead cells) was quantified by flow cytometry. ${ }^{23,24}$ As previously shown, ${ }^{22}$ monomeric and fibrillar $a$-syn were not toxic to the M17 cells over the concentration range from $20 \mathrm{nM}$ to $2 \mu \mathrm{M}$, whereas the addition of the crude mixture induced significant cell death after 4 days of treatment from concentrations as low as $200 \mathrm{nM}$ (Supplementary Figure S2). The conventional plate-based toxicity analyses agreed well with the single-cells experiments on the nontoxic nature of the monomeric $a-$ syn. $^{22}$ It is known that the monomeric, soluble form of $a$-syn is normally present in high concentration at the presynaptic terminals of the brain. ${ }^{21,25-27}$ In these physiological conditions, soluble monomers do not possess a neurotoxic effect. In contrast, most of the available evidences indicate that the aggregation of $a$-syn is essential for the induction of the pathological effects associated with PD. ${ }^{28-30}$

The subsequent step was the toxicity analyses of the M17 cells exposed to the different $a$-syn species at the single-cell level using the nanomotion assays. We used the micrometric motors of the AFM to attach few living cells (typically $3-5$ cells per experiment) to the surface of the sensor (Figure 1a) as previously described. ${ }^{16}$ We collected the deflections of the cantilever as a function of time using a conventional laser-based detection system and we used them to evaluate the activity of the cells (Figure 1b). The movements of the viable neuroblastomas caused an increase of the fluctuations (Supplementary Figure S3 and Supplementary Movie S1), while the inactivation or death of the cells resulted in a reduction of the movements of the sensor.

In order to exploit the peculiarities of the nanomotion sensor, we studied the cellular response of M17 neuroblastomas exposed to $a$-syn monomers, fibrils or to the crude mixture of species using a variety of time-resolved experimental pathways in a wide range of protein concentrations in solution ranging from 0.05 to $120 \mathrm{nM}$. Notably, in all cases, a single exposure to any of the amyloidogenic species did not induce cytotoxicity (described in details in the Supplementary Information 2, Supplementary Figure S4); this was a peculiarity of the nanomotion experimental setup. In order to investigate the possibility of cooperative effects and secondary processes in inducing cytotoxicity, ${ }^{29,31}$ we performed a two-step $a$-syn injection. A first injection of half of the target concentration was typically followed by a $1 \mathrm{~h}$ stabilization period and subsequently the concentration was increased to the target concentration.

In the case of $a$-syn monomers, the cellular viability of the M17 cells was only slightly affected by the exposure to the protein. Indeed, while the first dose had no measurable effect on the cell activity, the nanomotion signal remained constant for $>4 \mathrm{~h}$ after the injection of the second dose of proteins (that is, $>8 \mathrm{~h}$ from the start of the experiments, Figure 2a). Comparing the experiments involving a single-monomer injection to the results after the two-step procedure, we measured a $30-50 \%$ increase in the variance of the fluctuations after the second exposure, probably indicating a non-fatal cellular reaction to the proteins (Figure $2 a$ and Supplementary Figure S4a). This response could be detected only because of the sensitivity of the nanomotion sensor, since the concurrent optical investigation did not show any variation in the cell motility (Supplementary Movie S2). From the toxicity point of view, the cells were still alive and moving after $8 \mathrm{~h}$ from the start of the measurements, as in the control experiments (Supplementary Figure S3), indicating that the monomers did 

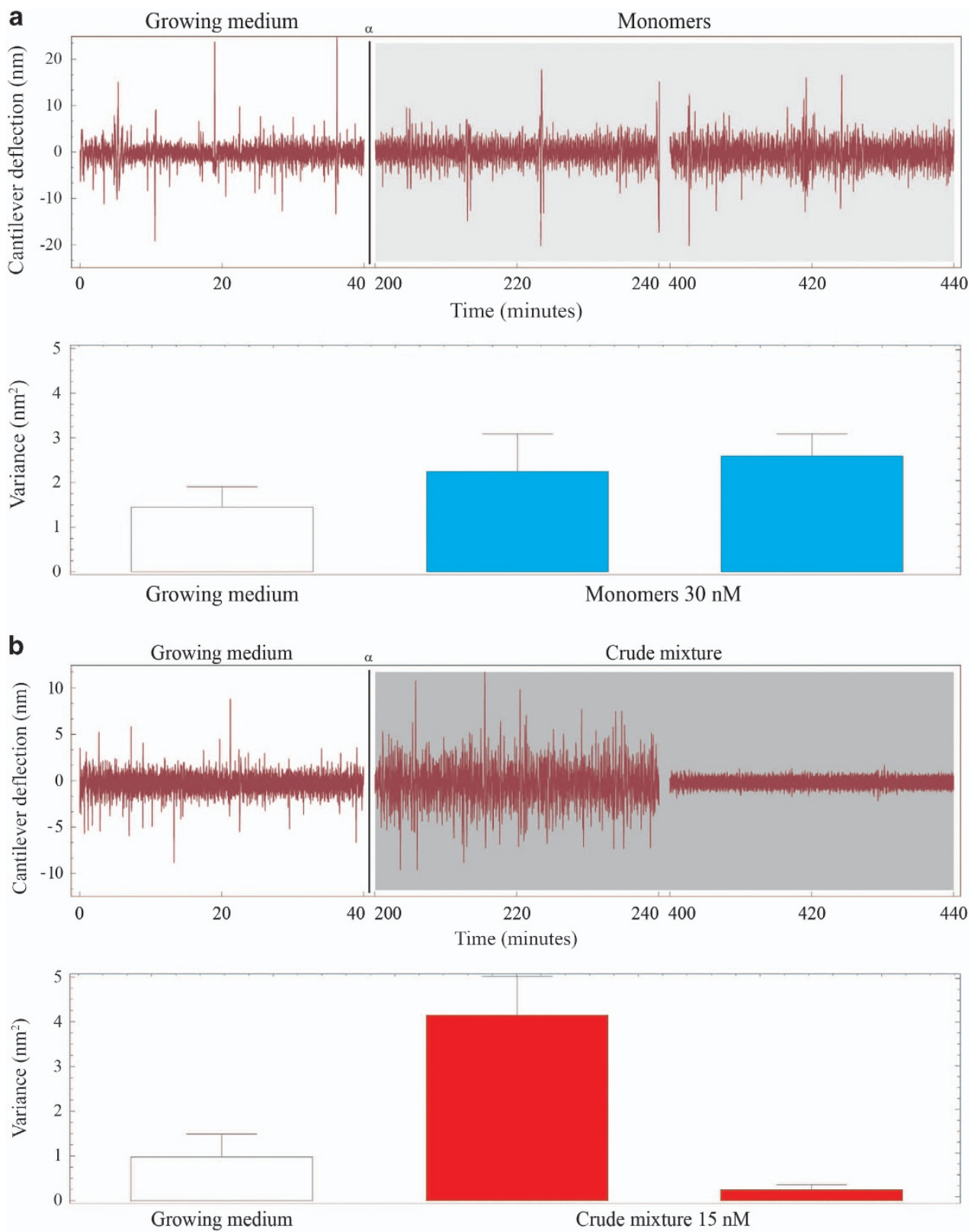

Figure 2. Nanomotion single-cell cytotoxicity studies. Typical nanomotion response of M17 neuroblastoma cells exposed to (a) monomeric $a$-syn and (b) the crude mixture of oligomers and fibrils. In both cases, the amyloid species were injected in a two-step procedure involving half dose $-1 \mathrm{~h}$ stabilization-half dose. The A point in the graph is relative to the last injection. After $4 \mathrm{~h}$ from the injection of the $a$-syn, the monomers do not cause a toxic reaction while the crude mixture had led the cells to death. More than 15 independent repeats were completed for each case. The histograms depict the average variance and the error bars indicate the variability of the variance over the chosen time-step.

not induce cytotoxic response in the M17 cells. Similarly, after several hours from the injection, the purified fibrillar $a$-syn samples did not induce any toxicity in the M17 cells, which were still active, both in the case of single injection (Supplementary Figure S4c) and when employing a double-injection protocol (Supplementary Figure S5). Next, we investigated the response of the neuroblastomas cells to the $a$-syn crude mixture delivered, as before, in two-step injection protocols with a $1 \mathrm{~h}$ stabilization period. The injection of the first half of the proteins did not modify the activity of the cells, which after $1 \mathrm{~h}$ were still exhibiting an activity comparable to the control single-step experiments (Supplementary Figure S4b). Remarkably, as depicted in Figure $2 \mathrm{~b}$, within the first $30 \mathrm{~min}$ from the second injection of the crude mixture, the cells increased their net fluctuations (the variance of the fluctuations increased up to 4-fold), indicating a strong cellular response to the crude $a$-syn mixture. The increase in fluctuations lasted in average about $2 \mathrm{~h}$ and ended abruptly with the cells undergoing cell death, as evidenced by the flatness of the nanomotion pattern, which indicated the absence of any cell-related activity. Moreover, the optical images showed that the cells increased their activity before swelling and, in many cases, exploding (Supplementary Movie S3). Overall, the addition of $a$-syn crude mixture in one single dose had no measurable effect on the M17 cells isolated on the cantilevers, while the two-step sequential injection induced a significant toxicity and induced a rapid cell death. Most of the cellular responses were undetectable using the conventional optical analyses, in which the M17 cells 

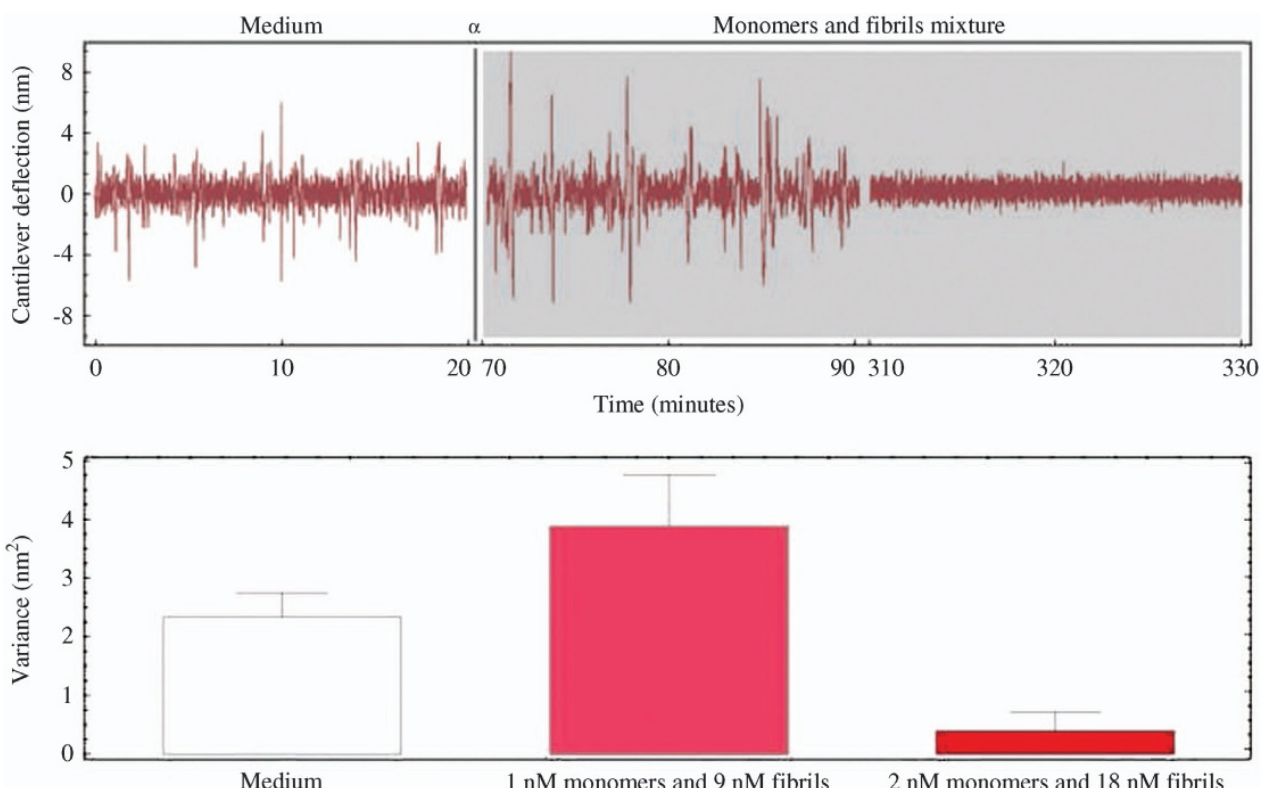

Figure 3. Nanomotion experiments using a mixture of $a$-syn monomers and fibrils. Using a two-step procedure, the M17 cells undergo a rapid cytotoxic reaction and, after $<4 \mathrm{~h}$, appear to have died. The histograms depict the average variance and the error bars indicate the variability of the variance over the chosen time-step.

appeared only to abruptly reduce their microscopic movements and die, often after some osmotic swelling.

The real-time monitoring and high sensitivity of the nanomotion sensor was fundamental to reveal the complex dynamic responses of the cells. Furthermore, in order to characterize fully the cytotoxic effect of the crude mixture as a function of the injected proteins concentration, we performed a series of nanomotion, dose-dependence experiments. These results show that even a small concentration of the mixture such as $2 \mathrm{nM}$ is capable to produce a cytotoxic effect on M17 cells isolated on a cantilever. (Supplementary Figure S6).

Finally, to allow a good comparison between these populationlevel experiments to the subsequent nanomotion sensor assays, we treated the cells in the wells with a two-step injection of monomers and crude mixture. At first, we injected half of the final chosen concentration of the $a$-syn and delayed the injection of the second dose of proteins for 1 or $24 \mathrm{~h}$. The conventional platebased toxicity analyses, both at single exposure and at two-step injection, agreed well with the single-cell experiments, confirming the cytotoxic nature of the aggregated mixture. This is in good agreement with previous results by our group ${ }^{22}$ and by other researchers, ${ }^{10,32-35}$ suggesting that the process of aggregation and fibril formation play central roles in amyloid toxicity (Supplementary Figure S7). ${ }^{36}$

The comparison between our experiments in multi-well plates and at the single-cell level highlighted the importance of the timing of the $a$-syn delivery in the determination of the cellular demise. The experiments on cantilevers required a two-step injection of $a$-syn crude mixture to produce cell death, while for the multi-well-based analyses, a single injection was sufficient to induce cytotoxicity. This is probably related to the experimental geometry: in the nanomotion setup, the insoluble material sediments quickly and is not continuously in contact with the cells throughout the incubation. Since the timescale for the multiwells experiments can be days, this sedimentation can lead to the formation of new amyloidogenic species, with unforeseen interactions with the cells. Notably, to deliver information on cellular response and measure cellular cytotoxicity, the nanomotion experiments required an experimental time (4-12 h) and a minimal crude mixture concentration ( $2 \mathrm{nM})$ up to two orders of magnitude smaller than the conventional plate analyses we performed ( 4 days, $200 \mathrm{nM}$ ). Also, this high sensitivity of the M17 cells in the nanomotion sensor assays can be tentatively attributed to the experimental geometry. The small number of cells on the sensor (at most 5 cells were present on the cantilever at the same time) and the space constraints imposed by the cantilever do not favor intercellular interactions and cell to cell communications, making the individual cells certainly more sensitive to their environment. ${ }^{37-39}$

\section{Cooperative cytotoxicity of amyloids species}

To understand better the cooperative role of monomeric and fibrillar species in the onset of cytotoxicity and to investigate the role of a two-step injection for the nanomotion experiments, we exposed the M17 cells either to a mixture of monomeric and fibrillar species (Figure 3 ) or to a sequential exposure to the two species in different order (Supplementary Table S1). In these experiments, we maintained constant the final concentration of the $a$-syn species at $20 \mathrm{nM}$. The monomers and fibrils mixture caused cytotoxicity in the M17 cells following a pattern very similar to the crude mixture case. A single exposure did not produce cell death, while a two-step exposure always led to cytotoxic response. On the other hand, the sequential injection of monomeric and fibrillar species always led to cell death and the relative concentration of monomers and fibrils had very little influence on the outcome. (Supplementary Table S1) This supports our results involving the crude mixture, since monomeric and fibrillar solutions always contain a certain amount of oligomeric species. Remarkably, the speed of the cell death depended on the sequence of exposure to each specific $a$-syn species. In fact, when the cells were exposed first to monomers and next to the fibrils, we observed a timing of the cytotoxic effect that was delayed compared to when fibrils where injected first (Supplementary Table S2). This finding support our previous data showing that $a$-syn fibrils that bind to the extracellular cell plasma membrane can serve as anchoring and nucleation points for the aggregation of extracellular monomeric $a$-syn at the cell plasma membrane, which disrupts the membrane permeability leading to cell death. ${ }^{22,29}$

Finally, to gain further insight into this phenomenon, and in particular to determine the localization of the interaction, we 

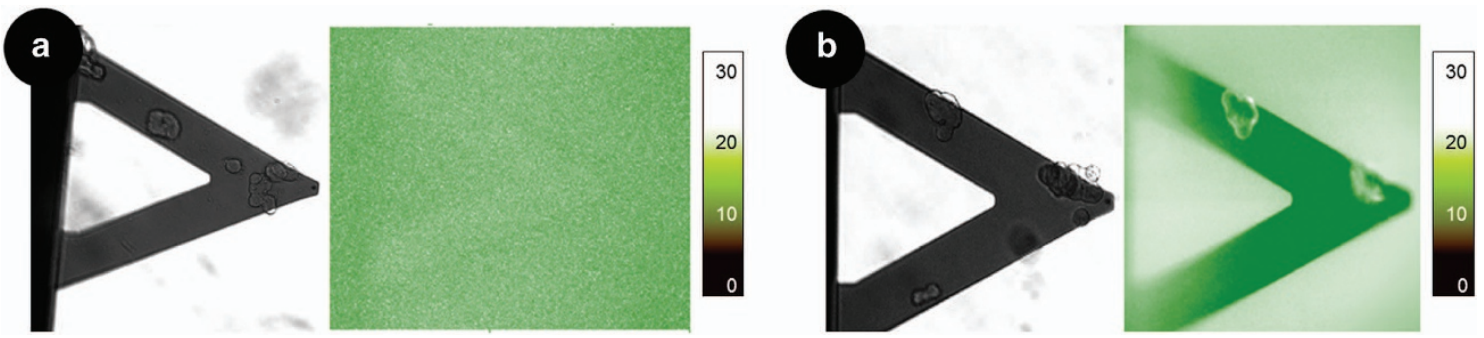

Figure 4. Localization of $a$-syn species. Optical and fluorescence images of M17 cells on a cantilever sensor exposed for $2 \mathrm{~h}$ to OG-tagged a-syn monomeric (a) and fibrillar (b) forms. The fibrils are clearly localized on the cell surface while the monomers do not appear to localize on the cells. (The nanomotion response is shown in Supplementary Figure S6).
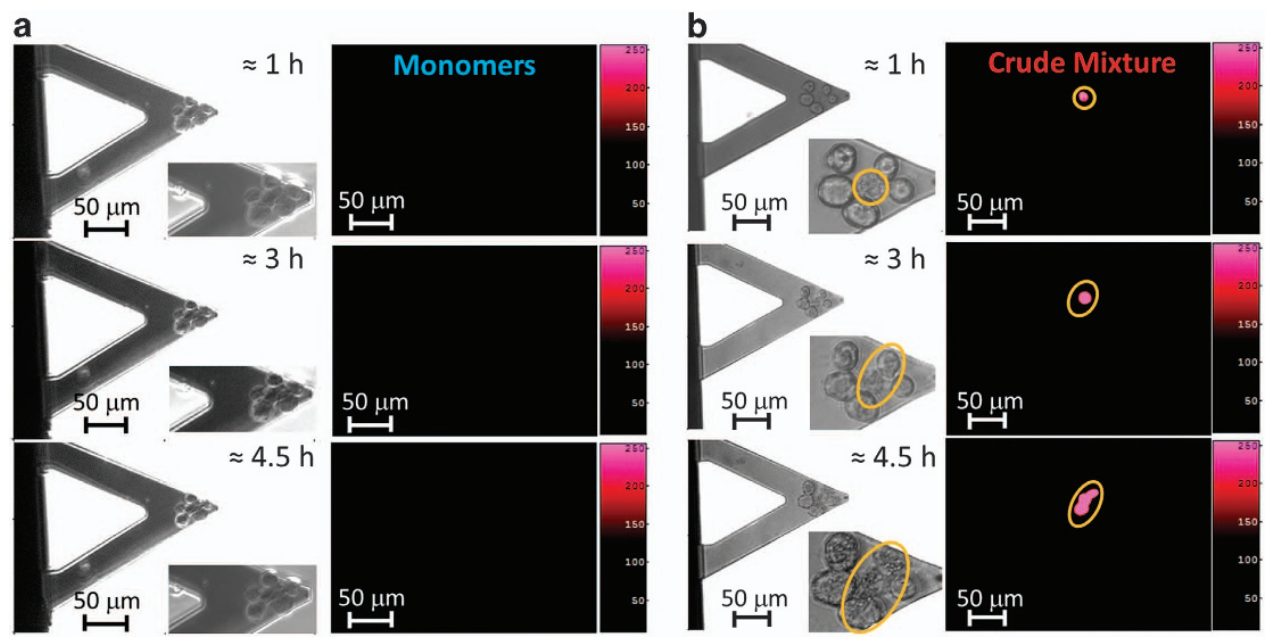

Figure 5. Internalization of the Ethidium homodimer III. Optical and fluorescence images of M17 cells in ETH III-rich medium after 1,3 and $4.5 \mathrm{~h}$ of incubation of cells with (a) $a$-syn monomers and (b) aggregated $a$-syn crude mixture at $0.12 \mu \mathrm{M}$.

exposed the M17 neuroblastomas to monomeric or fibrillar forms, which were previously tagged with Oregon Green fluorescent dye. For these experiments, we combined the nanomotion sensor with a fluorescence microscope to study simultaneously the viability and activity of the M17 cells and the localization of the highly homogeneous species $a$-syn proteins. As shown in Figure 4a, when exposed to the fluorescent monomeric form, the images do not suggest any specific protein localization on the M17 cells. On the other hand, when the cells were exposed to the fluorescent fibrils, we could observe enhanced fluorescence localized on the cell membrane, thus indicating that fibrillar $a$-syn has bonded on the surface of the M17 cells (Figure 4b). These results support the hypothesis of the cooperative interaction between multiple species of $a$-syn and M17 cells and especially the requirement for two-step $a$-syn injection in our configuration to induce the toxicity in M17 cells. ${ }^{32,40}$ Initially, the insoluble fibrillar species localize on the cell membrane and can act as seeds for the biological action of the soluble monomers and early oligomeric aggregates. $^{21}$

\section{Membrane permeabilization related cytotoxicity}

It has been suggested that the $a$-syn localization could affect the membrane permeabilization, thus inducing the cytotoxic action. In order to validate this hypothesis, we combined conventional fluorescence and nanoscale techniques to investigate the cellular response to extracellular $a$-syn at the single-cell level. We collected several cells on the cantilever sensor and exposed them to a buffer containing ethidium homodimer III (EtH III), a vital dye whose uptake is a marker of cell death and membrane integrity. After a first incubation period, we treated the cells either with $a$-syn monomers or with the crude mixture. The M17 cells exposed to $a$-syn monomers did not internalize any EtH III, even at the highest concentration of the proteins $(120 \mathrm{nM})$, whereas the exposure to the crude mixture led to a rapid and high internalization of EtH III, even while the cells were still viable. In fact, the time-resolved images showed that the level of the fluorescent dye accumulated over time in the M17 cells (Figure 5). Remarkably, the dye uptake corresponded very well with the increase of the cellular nanomotion, typical of the exposure to the crude mixture before the final cell death (Supplementary Figure S8).

These experiments support our hypothesis that membrane permeabilization is a key contributor to $a$-syn extracellular toxicity. The addition of the extracellular $a$-syn crude mixture, in the range between 2 and $120 \mathrm{nM}$ promotes the loss of plasma membrane integrity and the activation of a strong cellular response and final cytotoxicity, highlighted by the nanomotion experiments,. On the contrary, the monomers do not cause any cell permeabilization, well in agreement with the absence of any cytotoxicity. These results are in agreement with the assumption that the exposure to fibrillar forms or the process of $a$-syn fibrillization play central roles in $a$-syn-mediated neruoblastoma death via membrane permeabilization. 22,23

\section{DISCUSSION}

Investigating the response of mammalian cells to external chemical or physical stimuli is of paramount importance to study the cellular biology and the origin of human diseases, including neurodegenerative disorders such as Alzheimer's and Parkinson's diseases. In the present work, we employed a nanomotion 
detector to study at the single-cell level, the toxicity of monomeric and aggregated $a$-syn on M17 neuroblastoma cell lines. This phenotypic platform delivers cytotoxic responses very rapidly, almost two orders of magnitude faster than conventional platebased bulk toxicity assays and at near physiological concentrations, and has the ability to perform label-free analyses, making it the ideal complement to the well-known and established biological techniques at the population level. Furthermore, the nanomotion sensor can perform analyses on single or few cells, and requires a very small analysis chamber. The consequent use of much smaller volumes of buffers, proteins and reagents when compared to conventional bulk techniques, is of paramount importance when characterizing the effects of expensive drugs or chemicals. The comparison between nanomotion and conventional plate assays indicate that the nanomotion single-cell analyses have several advantages over conventional biological experiments, including faster screenings, real-time analyses of the cellular dynamics and time-resolved investigations.

Here, through a careful characterization of the proteins, we were able to demonstrate that the extracellular exposure of cells to a homogeneous monomeric or fibrillar solution of $a$-syn is not toxic, even at a single-cell level. On the other hand, we demonstrated that the cooperative action of the individually non-toxic species led to cytotoxicity and cell death, as in the case of a crude mixture containing residual monomeric and mainly oligomeric and fibrillar species. These results were in good agreement with the analyses at the population level using platebased toxicity assays. ${ }^{22}$ Furthermore, by combining the nanomotion information with fluorescence microscopy, we investigated the mechanism leading to cytotoxicity, showing that proteininduced loss of membrane integrity ultimately led cells to death. These results highlight the great potential of the nanomotion studies as a new fast and reliable analysis tool to study the molecular basis of amyloid toxicity in misfolding diseases at the single-cell level and, overall, to study a wide range of diseases and biological problems. This could pave the way to a new paradigm in the investigation of toxicity in cells and can become a tool to perform testing of protective drugs, to stop or delay the neurodegenerative toxicity mechanisms.

\section{MATERIALS AND METHODS}

Substrates, enzymes and reagents

All chemicals, phosphate buffered saline (PBS, pH 7.4), poly-L-lysine, Trypsin-EDTA, DMEM, glycine, F12 medium, fetal bovine serum (FBS), glutaraldehyde, all with analytical grade, were supplied by Life Technologies, (Carlsbad, CA, USA). The uranyl-formate solution was acquired from Electron Microscopy Sciences (Luzern, Switzerland), Ethidium Homodimer III (ETH III) from Promokine (Heidelberg, Germany) and Propidium lodide (PI) from Millipore (Schaffhausen, Switzerland).

Preparation and characterization of $a$-syn recombinant proteins: expression and purification of monomers

Human wild-type (WT) a-syn was subcloned in pT7-7 plasmid and transferred into the expression strain E.coli BL21. After its bacterial expression, $a$-syn was first purified by anion exchange chromatography $(\mathrm{AEC})$ and size exclusion chromatography (SEC) as described in detail by Fauvet et al. ${ }^{41}$ and then by reverse-phase HPLC (Waters 600 system) using a Cosmosil C4 preparative column $(20 \times 250 \mathrm{~mm}, 38048)$ with a linear gradient of $20-70 \%$ B (solvent $A: \mathrm{H}_{2} \mathrm{O} / 0.1 \%$ formic acid, solvent $\mathrm{B}$ : acetonitrile $/ 0.1 \%$ formic acid). The $a$-syn elution was monitored by UV absorbance at 214 and $280 \mathrm{~nm}$ and the mass was confirmed by MALDITOF-MS (Matrix-assisted laser desorption/ionization-Time of Flight), ESI-MS analysis (Electrospray ionization, Thermo Scientific, Carlsbad, CA, USA) and SDS-PAGE (Sodium dodecyl sulfate-Polyacrylamide gel electrophoresis). Acetonitrile was removed from the $a$-syn containing fractions using a rotary evaporator and the $a$-syn fractions were lyophilized and stored at $-20^{\circ} \mathrm{C}$ until further use. ${ }^{22}$ To ensure that the preparation of monomeric a-syn was free of preformed aggregates or oligomeric forms, as low as dimers, the stock solution was systematically filtered through a $100 \mathrm{kDa}$ filter.

Preparation and characterization of $a$-syn recombinant proteins: preparation of crude mixture

The $a$-syn crude mixture was generated incubating $800 \mu \mathrm{l}$ of a $45 \mu \mathrm{M}$ filtered (100 kDa filter) (Millipore) monomeric $a$-syn solution (50 mM Tris, $150 \mathrm{mM} \mathrm{NaCl}, \mathrm{pH} 7.5)$ under constant orbital agitation (400 rpm) at $37^{\circ} \mathrm{C}$ for 4 weeks. It is of particular importance to highlight that we chose not to sonicate our solutions, since this is known to lead to amyloid fibril breakage and this is suggested as a possible cause of enhanced toxicity. ${ }^{22}$

\section{Neuroblastoma cell culture}

The dopaminergic human neuroblastoma $\mathrm{M} 17$ cell line were cultured in humidified air under $5 \% \mathrm{CO}_{2}$ at $37^{\circ} \mathrm{C}$ in Dulbecco's Modified Eagle Medium and $50 \% \mathrm{~F}-12$ medium supplemented with $10 \%$ of inactivated FBS and $1 \%$ of Penicillin/Streptomycin solution. The M17 cells were detached from culture flask using Trypsin-EDTA for $5 \mathrm{~min}$ at $37^{\circ} \mathrm{C}$, before further use.

\section{The nanomotion experiments}

The nanomotion analyses were carried out using a Nanowizard III atomic force microscope (JPK, Berlin, Germany), using commercial silicon nitride, micro-cantilevers, with a nominal spring constant of $0.12 \mathrm{~N} / \mathrm{m}$ (DNP-10 Bruker). The microscope was equipped with a custom liquid cell ${ }^{42}$ and coupled with an Axiovert $X$ optical microscope (Zeiss Microscopy, Germany). All the optical images were collected using a standard 40x objective in the phase-contrast modality through a Progres MFCool digital camera (Jenoptik, Germany). The time-dependent fluctuations of the sensor, linked to the metabolic activity of the biological specimens, were recorded through the JPK control software and we performed the data analysis using a custom software written in LabView (National Instruments, USA). The cantilever fluctuations were recorded with a sampling frequency of $10 \mathrm{kHz}$.

\section{Temperature control}

The temperature of the analysis chamber was controlled using a custom petri-cell heater, which was calibrated to ensure the cells were kept at $37^{\circ} \mathrm{C}$ throughout the entire experiment. Before inserting the cells in the analysis chamber, the system was first stabilized; less than 30 min were needed to obtain a perfectly stable cantilever. All the solutions were left to thermalize for minimally $1 \mathrm{~h}$ prior to injection in the analysis chamber. The temperature of all the injected media was controlled just before the injection using a bimetallic temperature sensor (DT120, Rüeger, Switzerland). This ensured that the temperature throughout the entire experimental analysis was constant within $0.1^{\circ} \mathrm{C}$.

\section{Characterization of the nanomotion sensor}

Each cantilever was preliminarily characterized in the fluid environment using the thermal fluctuation method ${ }^{43,44}$ in order to calculate its resonance frequency and the effective spring constant (which were in good agreement with the nominal values). For each experiment, the thermal fluctuation analysis was performed before and after the attachment of the cells, in order to determine any variations of the mechanical properties of the sensor throughout each experiment.

\section{Functionalization protocol and cell fishing}

In our previous works, we have highlighted how the choice of the correct functionalization of the sensor surface is fundamental to obtain the best immobilization efficiency. ${ }^{16}$ To ensure a good attachment of the neuroblastoma cells on the cantilever the sensor was covered with a droplet of $10 \%$ (vol/vol) poly-L-lysine and left to incubate for $30 \mathrm{~min}$ at room temperature. Next, the sensor was rinsed thoroughly using ultrapure water and transferred it to the analysis chamber, which was flushed with a cellular nourishing buffer containing a small concentration of live cells. Then, we used the AFM coarse and fine movement capabilities to collect some cells, ${ }^{45}$ attaching them near the apical region of the cantilever (typically, 3-5 individual cells were attached per experiment). Once the cells were firmly attached to the sensor, the tip was retracted $\sim 100 \mu \mathrm{m}$ from the surface and the nanomotion experiments were carried out. 
The nanomotion detector

The setup of the nanomotion detection experiments is described in detail in our pioneering works ${ }^{15,16}$ and is depicted in Figure 1. Each nanomotion experiment started with $1 \mathrm{~h}$ stabilization period, which was necessary to verify the viability and the complete adhesion of the cells on the sensor. Throughout this first period, the sensor was immersed in growing buffer and we monitored its fluctuations as a function of time, while recording every $20 \mathrm{~s}$ an optical image of the cells. The fluctuations of the sensor were used to investigate the nanometer-scale movements of the adhering cells, while the optical images showed their micron-sized evolution on the cantilever surface. These measurements reflected the basal metabolic activity of the cells. While collecting the nanomotion signal, we recorded time-lapse videos of the cells on the sensor, to check their attachment, their healthiness and to monitor at the micrometer-scale the individual cell movements during the time of the experiment.

After the first stabilization period, we exploited the input-output tubes of the analysis chamber to introduce an enriched medium containing the $a$-syn monomers or crude mixture. We performed several experiments with monomer, fibrils crude mixture protein concentration ranging between 0.05 and $120 \mathrm{nM}$. The stability of the liquid in the analysis chamber is crucial in our experimental setup. Therefore, after injecting the medium, we waited at least $5 \mathrm{~min}$ to ensure that the liquid had stabilized before starting the measurement. The injection of new media in the analysis chamber was performed at very slow rates $(<8 \mu \mathrm{l} / \mathrm{s})$, always verifying through the optical images that the samples on the cantilever sensor were unaffected by the flow. Thus, we were capable of monitoring the movements of the cells and their viability for more than $12 \mathrm{~h}$, to identify the cellular response to the $a$-syn treatment.

To evaluate all the experimental results, we analyzed statistically the fluctuations by calculating their variance and we repeated each experiment at least 3 times to ensure a good repeatability of the results.

Investigation of cell death by dye exclusion method in M17 cells on the nanomotion sensor

The M17 cells were picked up on the cantilever and, after the stabilization period, they were incubated in the growing medium added with $1 \mu \mathrm{M}$ of Ethidium Homodimer III (EtH III), a membrane impermeant dye, which enters only in cells with damaged plasma membranes.

To monitor the capacity of the M17 cells to internalize the vital dye, we used a Zeiss Axiovert Z1 system to acquire optical and fluorescence images of the cells on the cantilever. After 15 min of incubation, we collected the baseline images of the neuroblastoma cells (time-point 0 ). Next, we added the extracellular monomeric $a$-syn, or $a$-syn crude mixture (at $120 \mathrm{nM}$ in both cases) to the cells. The internalization of the EtH III into the cells was recorded by collecting, every $10 \mathrm{~min}$, optical and fluorescence images of the cells for up to $5 \mathrm{~h}$. Each image was acquired with an exposition time of $2 \mathrm{~s}$. In parallel, we monitored the cellular response in terms of nanomotion, in order to understand if the presence of the fluorescent dye had influenced the cells. All the optical images were analyzed by means of SPIP software (Image Metrology, Hørsholm, Denmark).

Quantification of cell death by dye exclusion method in M17 cells by flow cytometry

The M17 cell lines were plated in 24 wells plates. After $24 \mathrm{~h}$, they were treated with extracellular monomeric $a$-syn, or $a$-syn crude mixture or Tris buffer (50 mM Tris, $150 \mathrm{mM} \mathrm{NaCl}, \mathrm{pH} 7.5$ ) as negative control. Cell death was quantified by the vital dye exclusion method using Propidium lodide $(\mathrm{Pl})$, a vital dye that enters only in cells with compromised membranes. Briefly, the supernatant and the adherent cells were harvested and collected in FACS tube. After 5 min of centrifugation at $500 \mathrm{~g}$, pelleted cells were resuspended in PBS and $\mathrm{PI}$ was added to the cells at a final concentration of $2 \mu \mathrm{g} / \mathrm{ml}$. Cells were then analyzed by flow cytometry using Accuri C6 (BD Biosciences, Allschwil, Switzerland) and FlowJo software (Treestar, Ashland, OR, USA).

\section{Circular dichroism}

Samples were analyzed at room temperature (RT) using a Jasco J-815 CD spectrometer. An average of 10 spectra was collected in the range of $190-250 \mathrm{~nm}$ using a 1.0-mm-optical-pathlength quartz cuvette. The data points were acquired every $0.2 \mathrm{~nm}$ in the continuous scanning mode at a speed of $50 \mathrm{~nm} / \mathrm{min}$ with a digital integration time of $2 \mathrm{~s}$ and a bandwidth of $1 \mathrm{~nm}$. The processed spectra were obtained by subtracting the baseline signal due to the water and cell contribution from the protein spectra.

\section{Thioflavin T}

Thioflavin T (ThT) fluorescence reading was measured for $a$-syn at $45 \mu \mathrm{M}$, diluted at $1.5 \mu \mathrm{M}$ with a ThT concentration of $10 \mu \mathrm{M}$, in a $70 \mu \mathrm{l}$ solution with $\mathrm{pH} 8.5$ buffer containing $50 \mathrm{mM}$ glycine. A Bucher Analyst AD plate reader was used to measure ThT fluorescence at an excitation wavelength of $450 \mathrm{~nm}$ and an emission wavelength of $485 \mathrm{~nm}$. Aliquots taken at different time points were measured in triplicate.

\section{Electron microscopy analyses}

A $10 \mu \mathrm{l}$ droplet of the sample was deposited on Formvar/carbon-coated 200-mesh copper grids (Electron Microscopy Sciences). The grids were washed twice with $5 \mu \mathrm{l}$ of ultrapure water and then stained twice with $5 \mu \mathrm{l}$ of an aqueous $2 \% \mathrm{w} / \mathrm{v}$ uranyl-formate solution and then vacuum-dried from the edges of the grids. Samples were imaged using a Tecnai Spirit BioTWIN electron microscope (TEM) operated at $80 \mathrm{kV}$ with a $\mathrm{LaB}_{6}$ source.

\section{Atomic force microscopy imaging}

We characterized the morphology of the different $a$-syn states by Atomic Force Microscopy (AFM). We performed these investigations on positively functionalized mica. After the cleaving, the mica substrate, was incubated with a $10 \mu \mathrm{l}$ drop of $0.05 \%(\mathrm{v} / \mathrm{v})$ APTES in Milli-Q water for $1 \mathrm{~min}$ at ambient temperature, rinsed with Milli-Q water and then dried by the passage of a gentle flow of gaseous nitrogen. AFM samples preparation was realized at room temperature by deposition of a $10 \mu \mathrm{l}$ aliquot of full concentrated solution on the surface for $5 \mathrm{~min}$. AFM images were realized in ambient condition by means of a Park NX10 (Park, USA) operating in true noncontact mode and equipped with a silicon tip (Nanosensor, PPP-NCHR, $40 \mathrm{Nm}^{-1}$ ) with a nominal radius of $7 \mathrm{~nm}$. Image flattening was applied to each image and the analysis was performed using SPIP software.

\section{Statistical analysis}

For the flow cytometry experiments to determine cell death, we performed a one-way ANOVA test followed by a Tukey-Kramer post hoc test. The data were regarded as statistically significant at $P<0.05$ based on the TukeyKramer post hoc test.

Regarding the single-cell analyses, each nanomotion experiment described in this work was the result of a series of least three independent experiments. The representative nanomotion graphs and variance measurements presented in the manuscript are representative of the general behavior of triplicates, which showed all the same behavior. In all graphs, we presented the data with the relative standard deviation. The times-to-death graphs were averaged over minimally three independent experiments.

Data and materials availability

All the data are freely available on request from the authors.

\section{ACKNOWLEDGEMENTS}

We especially thank the following members of the Lashuel group; Nadine Ait Bouziad for help with collecting the TEM images of the fibrils shown in Figure S1; Nathalie Jordan for preparing the primary culture; and Celine Vocat and Filip Vercruysse for preparing some of the $a$-syn recombinant protein used in this study. We also thank Darren Moore for providing the M17 cells and SH-S5Y5 cell lines. Longo would like to thank, Benedetta Mannini, Simone Dinarelli and Marco Girasole for helpful comments. This work was supported by the Swiss National Foundation for Science, grant number 152958.

\section{AUTHOR CONTRIBUTIONS}

FSR, GD and GL conceived and planned the experiments. A-LM. prepared the cells and performed the bulk experiments. FSR. prepared the proteins and conducted the characterizations. FSR and GL performed the nanomotion and fluorescence experiments and analyzed the data. FSR and GL wrote the manuscript. All the authors commented the manuscript. 


\section{COMPETING INTERESTS}

The authors declare no competing financial interest. The nanomotion sensor has been patented n. PCT/WO2013054311 with an international extension in 2014.

\section{PUBLISHER'S NOTE}

Springer Nature remains neutral with regard to jurisdictional claims in published maps and institutional affiliations.

\section{REFERENCES}

1 Levsky JM, Singer RH. Gene expression and the myth of the average cell. Trends Cell Biol 2003; 13: 4-6.

2 Mannini B, Mulvihill E, Sgromo C, Cascella R, Khodarahmi R, Ramazzotti M et al. Toxicity of Protein Oligomers Is Rationalized by a Function Combining Size and Surface Hydrophobicity. ACS Chem Biol 2014; 9: 2309-2317.

3 Zampagni M, Cascella R, Casamenti F, Grossi C, Evangelisti E, Wright D et al. A comparison of the biochemical modifications caused by toxic and non-toxic protein oligomers in cells. J Cell Mol Med 2011; 15: 2106-2116.

4 Shah P, Kaushik A, Zhu X, Zhang C, Li C-Z. Chip based single cell analysis for nanotoxicity assessment. Analyst 2014; 139: 2088-2098.

5 Luna E, Luk KC. Bent out of shape: alpha-Synuclein misfolding and the convergence of pathogenic pathways in Parkinson's disease. FEBS Lett 2015; 589: 3749-3759.

6 Brettschneider J, Tredici KD, Lee VMY, Trojanowski JQ. Spreading of pathology in neurodegenerative diseases: a focus on human studies. Nat Rev Neurosci 2015; 16: 109-120.

7 Irwin DJ, Lee VM, Trojanowski JQ. Parkinson's disease dementia: convergence of alpha-synuclein, tau and amyloid-beta pathologies. Nat Rev Neurosci 2013; 14: 626-636.

8 Lashuel HA, Overk CR, Oueslati A, Masliah E. The many faces of [alpha]-synuclein: from structure and toxicity to therapeutic target. Nat Rev Neurosci 2013; 14: 38-48.

9 Goedert M, Spillantini MG, Del Tredici K, Braak H. 100 years of Lewy pathology. Nat Rev Neurol 2013; 9: 13-24.

10 Marques O, Outeiro TF. Alpha-synuclein: from secretion to dysfunction and death. Cell Death Dis 2012; 3: e350.

11 Lee H-J, Bae E-J, Lee S-J. Extracellular [alpha]-synuclein[mdash]a novel and crucial factor in Lewy body diseases. Nat Rev Neurol 2014; 10: 92-98.

12 Luk KC, Kehm V, Carroll J, Zhang B, O'Brien P, Trojanowski JQ et al. Pathological alpha-synuclein transmission initiates Parkinson-like neurodegeneration in nontransgenic mice. Science 2012; 338: 949-953.

13 Guo JL, Lee VMY. Cell-to-cell transmission of pathogenic proteins in neurodegenerative diseases. Nat Med 2014; 20: 130-138.

14 Recasens A, Dehay B, Bové J, Carballo-Carbajal I, Dovero S, Pérez-Villalba A, Fernagut $\mathrm{PO}$ et al. Lewy body extracts from Parkinson disease brains trigger $a$-synuclein pathology and neurodegeneration in mice and monkeys. Ann Neurol 2014; 75: 351-362.

15 Longo G, Alonso-Sarduy L, Rio LM, Bizzini A, Trampuz A, Notz J et al. Rapid detection of bacterial resistance to antibiotics using AFM cantilevers as nanomechanical sensors. Nat Nano 2013; 8: 522-526.

16 Kasas S, Ruggeri FS, Benadiba C, Maillard C, Stupar P, Tournu H et al. Detecting nanoscale vibrations as signature of life. Proc Natl Acad Sci 2015; 112: 378-381.

17 Lissandrello C, Inci F, Francom M, Paul MR, Demirci U, Ekinci KL. Nanomechanical motion of Escherichia coli adhered to a surface. Appl Phys Lett 2014; 105: 113701.

18 Aghayee S, Benadiba C, Notz J, Kasas S, Dietler G, Longo G. Combination of fluorescence microscopy and nanomotion detection to characterize bacteria. $J$ Mol Recognit 2013; 26: 590-595.

19 Alonso-Sarduy L, De Los Rios P, Benedetti F, Vobornik D, Dietler G, Kasas S et al. Real-Time Monitoring of Protein Conformational Changes Using a NanoMechanical Sensor. PLoS One 2014; 9: e103674.

20 Ruggeri FS, Adamcik J, Jeong JS, Lashuel HA, Mezzenga R, Dietler G. Influence of the $\beta$-sheet content on the mechanical properties of aggregates during amyloid fibrillization. Angewandte Chemie 2015; 127: 2492-2496.

21 Khalaf O, Fauvet B, Oueslati A, Dikiy I, Mahul-Mellier AL, Ruggeri FS et al. The H50Q mutation enhances alpha-synuclein aggregation, secretion, and toxicity. J Biol Chem 2014; 289: 21856-21876.

22 Mahul-Mellier AL, Vercruysse F, Maco B, Ait-Bouziad N, De Roo M, Muller D et al. Fibril growth and seeding capacity play key roles in [alpha]-synuclein-mediated apoptotic cell death. Cell Death Differ 2015; 22: 2107-2122.

23 Riccardi C, Nicoletti I. Analysis of apoptosis by propidium iodide staining and flow cytometry. Nat Protoc 2006; 1: 1458-1461.
24 Macklis JD, Madison RD. Progressive incorporation of propidium iodide in cultured mouse neurons correlates with declining electrophysiological status: a fluorescence scale of membrane integrity. J Neurosci Methods 1990; 31: 43-46.

25 Davidson WS, Jonas A, Clayton DF, George JM. Stabilization of alpha-synuclein secondary structure upon binding to synthetic membranes. J Biol Chem 1998; 273: 9443-9449.

26 McLean PJ, Kawamata H, Ribich S, Hyman BT. Membrane association and protein conformation of alpha-synuclein in intact neurons. Effect of Parkinson's diseaselinked mutations. J Biol Chem 2000; 275: 8812-8816.

27 Eliezer D, Kutluay E, Bussell R, Browne G. Conformational properties of alphasynuclein in its free and lipid-associated states. J Mol Biol 2001; 307: 1061-1073.

28 Lansbury PT, Lashuel HA. A century-old debate on protein aggregation and neurodegeneration enters the clinic. Nature 2006; 443: 774-779.

29 Knowles TP, Vendruscolo M, Dobson CM. The amyloid state and its association with protein misfolding diseases. Nat Rev Mol Cell Biol 2014; 15: 384-396.

30 Duda JE, Lee VMY, Trojanowski JQ. Neuropathology of synuclein aggregates. J Neurosci Res 2000; 61: 121-127.

31 Jeong JS, Ansaloni A, Mezzenga R, Lashuel HA, Dietler G. Novel mechanistic insight into the molecular basis of amyloid polymorphism and secondary nucleation during amyloid formation. J Mol Biol 2013; 425: 1765-1781.

32 Volpicelli-Daley LA, Luk KC, Patel TP, Tanik SA, Riddle DM, Stieber A et al. Exogenous alpha-synuclein fibrils induce Lewy body pathology leading to synaptic dysfunction and neuron death. Neuron 2011; 72: 57-71.

33 Luk KC, Song C, O'Brien P, Stieber A, Branch JR, Brunden KR et al. Exogenous alpha-synuclein fibrils seed the formation of Lewy body-like intracellular inclusions in cultured cells. Proc Natl Acad Sci USA 2009; 106: 20051-20056.

34 Di Giovanni S, Eleuteri S, Paleologou KE, Yin G, Zweckstetter M, Carrupt PA et al. Entacapone and tolcapone, two catechol O-methyltransferase inhibitors, block fibril formation of alpha-synuclein and beta-amyloid and protect against amyloidinduced toxicity. J Biol Chem 2010; 285: 14941-14954.

35 El-Agnaf OM, Jakes R, Curran MD, Middleton D, Ingenito R, Bianchi E et al. Aggregates from mutant and wild-type alpha-synuclein proteins and NAC peptide induce apoptotic cell death in human neuroblastoma cells by formation of beta-sheet and amyloid-like filaments. FEBS Lett 1998; 440: 71-75.

36 Jan A, Adolfsson O, Allaman I, Buccarello AL, Magistretti PJ, Pfeifer A et al. Abeta42 neurotoxicity is mediated by ongoing nucleated polymerization process rather than by discrete Abeta42 species. J Biol Chem 2011; 286: 8585-8596.

37 Payne S, You L. Engineered cell-cell communication and its applications. Adv Biochem Eng Biotechnol 2014; 146: 97-121.

38 Snijder B, Pelkmans L. Origins of regulated cell-to-cell variability. Nat Rev Mol Cell Biol 2011; 12: 119-125.

39 Rokas A. The Origins of Multicellularity and the Early History of the Genetic Toolkit For Animal Development. Annu Rev Genet 2008; 42: 235-251.

40 Volpicelli-Daley LA, Luk KC, Lee VM. Addition of exogenous alpha-synuclein preformed fibrils to primary neuronal cultures to seed recruitment of endogenous alpha-synuclein to Lewy body and Lewy neurite-like aggregates. Nat Protoc 2014; 9: 2135-2146.

41 Fauvet B, Mbefo MK, Fares MB, Desobry C, Michael S, Ardah MT et al. alphasynuclein in central nervous system and from erythrocytes, mammalian cells, and escherichia coli exists predominantly as disordered monomer. J Biol Chem 2012; 287: 15345-15364.

42 Kasas S, Radotic K, Longo G, Saha B, Alonso-Sarduy L, Dietler G et al. A universal fluid cell for the imaging of biological specimens in the atomic force microscope. Microsc Res Tech 2013; 76: 357-363.

43 Florin E, Moy V, Gaub H. Adhesion forces between individual ligandreceptor pairs. Science 1994; 264: 415-417.

44 Hutter JL, Bechhoefer J. Calibration of Atomic-Force Microscope Tips. Rev Sci Instrum 1993; 64: 1868-1873.

45 Benoit M, Gabriel D, Gerisch G, Gaub HE. Discrete interactions in cell adhesion measured by single-molecule force spectroscopy. Nat Cell Biol 2000; 2: 313-317.

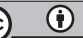

This work is licensed under a Creative Commons Attribution 4.0 International License. The images or other third party material in this article are included in the article's Creative Commons license, unless indicated otherwise in the credit line; if the material is not included under the Creative Commons license, users will need to obtain permission from the license holder to reproduce the material. To view a copy of this license, visit http://creativecommons.org/licenses/ by/4.0/

(c) The Author(s) 2017

Supplementary Information accompanies the paper on the Cell Death Discovery website (http://www.nature.com/cddiscovery) 